\title{
Eigenvalue-Based Time Delay Estimation of Repetitive Biomedical Signals
}

\author{
Pablo Laguna ${ }^{\mathrm{a}, \mathrm{g}}$, Ainara Garde ${ }^{\mathrm{b}}$, Beatriz F. Giraldo ${ }^{\mathrm{c}, \mathrm{d}, \mathrm{g}}$, Olivier Meste ${ }^{\mathrm{e}}$, \\ Raimon Janéc, $, \mathrm{d}, \mathrm{g}$, Leif Sörnmo $^{\mathrm{f}}$ \\ ${ }^{a}$ Biomedical Signal Interpretation \& Computational Simulation (BSICoS), Aragón Institute of Engineering \\ Research (I3A), Zaragoza University, Zaragoza, Spain. \\ ${ }^{b}$ Faculty of Electrical Engineering, Mathematics and Computer Science, University of Twente, Enschede, \\ The Netherlands. \\ ${ }^{c}$ Department of Automatic Control (ESAII), Universitat Politècnica de Catalunya (UPC)-Barcelona Tech, \\ Barcelona, Spain. \\ ${ }^{d}$ Institute for Bioengineering of Catalonia (IBEC), The Barcelona Institute of Science and Technology, \\ Barcelona, Spain. \\ ${ }^{e}$ Laboratoire d'Informatique, Signaux et Systèmes, Universite de Nice, Sophia Antipolis, France. \\ ${ }^{f}$ Department of Biomedical Engineering and Center for Integrative Electrocardiology (CIEL), Lund \\ University, Lund, Sweden. \\ ${ }^{g}$ Centro de Investigacíon Biomédica en Red de Bioingeniería, Biomateriales y Nanomedicina \\ (CIBER-BBN), Spain.
}

\begin{abstract}
The time delay estimation problem associated with an ensemble of misaligned, repetitive signals is revisited. Each observed signal is assumed to be composed of an unknown, deterministic signal corrupted by Gaussian, white noise. This paper shows that maximum likelihood (ML) time delay estimation can be viewed as the maximization of an eigenvalue ratio, where the eigenvalues are obtained from the ensemble correlation matrix. A suboptimal, one-step time delay estimate is proposed for initialization of the ML estimator, based on one of the eigenvectors of the inter-signal correlation matrix. With this approach, the ML estimates can be determined without the need for an intermediate estimate of the underlying, unknown signal. Based on respiratory flow signals, simulations show that the variance of the time delay estimation error for the eigenvalue-based method is almost the same as that of the ML estimator. Initializing the maximization with the one-step estimates, rather than using the ML estimator alone,
\end{abstract}

Email addresses: laguna@unizar.es (Pablo Laguna ), a.gardemartinez@utwente.nl (Ainara Garde), beatriz.giraldo@upc.edu (Beatriz F. Giraldo), olivier.meste@unice.fr (Olivier Meste), raimon. jane@upc. edu (Raimon Jané), leif.sornmo@bme. lth. se (Leif Sörnmo) 
the computation time is reduced by a factor of $5^{M}$ when using brute force maximization ( $M$ denoting the number of signals in the ensemble), and a factor of about 1.5 when using particle swarm maximization. It is concluded that eigenanalysis of the ensemble correlation matrix not only provides valuable insight on how signal energy, jitter, and noise influence the estimation process, but it also leads to a one-step estimator which can make the way for a substantial reduction in computation time.

Keywords: biomedical signals, time delay estimation, eigenanalysis, ensemble analysis.

\section{Introduction}

Time delay estimation represents a classical problem in biomedical signal processing, relevant for many applications such as high-resolution ECG, event-related brain potentials, conduction estimation in electromyography, and respiratory flow signals. In these applications, ensemble averaging, or some of its many variants [2], is applied to achieve noise reduction. To avoid distortion in the averaging process, prior alignment of the ensemble with similar-shaped signals is required. Another application is to sort spikes originating from the extracellular activity of different neurons; time alignment is then an important preprocessing step which ensures that spikes with similar shape

10 are assigned to the same cluster [3, 4]. Applications of high-resolution time alignment include the estimation of muscle fiber conduction velocity [5], the analysis of PR interval variability in the ECG observed during exercise and recovery [6], and the analysis of QT interval adaptation associated with changes in heart rate [7].

Despite the long-standing interest in time alignment, very few methods have been proposed which are inherently designed to jointly align the delayed signals of an ensemble. Rather, methods for pairwise time alignment of signals are employed as the basic operation, performed either in the time [8,3,9,10], frequency [11, 12, 13, 14], or scale domain [15]. The classical method for joint alignment of an ensemble is the Woody method [16], where the time delays are estimated by computing the crosscorrelation between each delayed signal and a reference signal ("the matched filter"), and finding the location of the maximum. The initial reference signal is taken as the 
ensemble average of the unaligned signals, then updated iteratively as new time delay estimates become available; this iterative procedure is terminated when the estimates no longer change. Although often used, the Woody method is empirical in nature as it does not ensure optimality in any sense.

Several papers have addressed the limitations of the Woody method by expanding it to handle colored noise [17], multicomponent signals [18, 9, 19], and nonlinear time scales $[20,21]$, whereas the problem of joint optimal time delay estimation remains largely unaddressed. However, Cabasson and Meste [22] derived the joint maximum likelihood (ML) estimator of the time delays, assuming that each observed signal is composed of an unknown, deterministic signal with unknown time delay and additive, Gaussian, white noise. Based on the structure of the log-likelihood function, the authors proposed an iterative procedure being identical to the Woody method, except that the intermediate ensemble average does not involve the signal subject to time delay estimation. Simulation results showed that, for small ensemble sizes ( $<25$ signals $)$, the resulting time delay estimates exhibited lower error variance than did those of the Woody method, whereas the error variances were virtually the same for larger sizes. However, the method in [22] does not guarantee optimality for the given model assumptions as the log-likelihood function is not subject to global maximization with respect to the time delays. Later, in [23], it was considered the joint time delay ML estimation for cases with coloured time delay distribution, deriving expressions that reduce to those in [22] when no correlation exits.

This paper introduces a novel approach to time alignment in which the eigenvalues of the intra-signal sample correlation matrix of an ensemble with delayed signals are explored. The method is based on the observation that a misaligned ensemble is associated with eigenvalues which depend on the misalignment variance. The ratio of the largest eigenvalue and the sum of the remaining eigenvalues is maximized when the ensemble is optimally aligned, and therefore the maximization of this ratio is proposed as a time delay estimator. In contrast to the iterative solution of the ML estimator [22], the eigenvalue-based estimator operates without the need for an intermediate estimate of the deterministic signal. It is shown that the ML estimator can be implemented by maximizing the first eigenvalue of this matrix, yielding results identical to those 
of the eigenvalue ratio estimator. The eigenvalue-based approach paves the way for a fast one-step estimator based on the second eigenvector of the inter-signal correlation matrix, well-suited for initializing the maximization required in the ML or the eigenvalue-based estimators. By pursuing eigenanalysis of the ensemble, new insight is provided on how signal energy, jitter, and noise influence the estimation process.

The present paper is organized as follows. Section 2 presents the basic idea of time alignment, provides an interpretation of the alignment criterion, and describes the maximization procedure. Section 3 details the simulation setup considered for performance evaluation. Section 4 presents the data used to test the method on a real scenario, followed by sections with results and discussion.

\section{Methods}

\subsection{Signal model and correlation matrix formulation}

In time alignment of repetitive biomedical signals, each one of the $M$ observed signal $x_{i}(n)$ of the ensemble is often modeled by $[1,2]$

$$
x_{i}(n)=s\left(n-\theta_{i}\right)+v_{i}(n), \quad n=0, \ldots, N-1 ; i=1, \ldots, M,
$$

where $s(n)$ is an unknown, deterministic signal with energy $E_{s}, \theta_{i}$ is a random, zeromean, symmetrically-distributed, integer-valued time delay with variance $\sigma_{\theta}^{2}$, and $v_{i}(n)$ is zero-mean, Gaussian, white noise with variance $\sigma_{v}^{2} ; \theta_{i}$ and $v_{i}(n)$ are assumed to be uncorrelated. The relevance of these assumptions for biomedical signals is discussed in Section 6. The compact support subinterval of $s\left(n-\theta_{i}\right), n=n_{o}, \ldots, n_{e}$, is assumed to be contained in the interval $[0, N-1]$ :

$$
s\left(n-\theta_{i}\right) \neq 0, n \in\left[0+\Delta_{\max }, N-\Delta_{\max }\right],
$$

for $\theta_{i}$ under consideration. The margin $\Delta_{\max }$ is introduced to guarantee compact support in $[0, N-1]$ also after time alignment. The signal ensemble is represented by the column matrix

$$
\mathbf{X}=\left[\begin{array}{lll}
\mathbf{x}_{1} & \cdots & \mathbf{x}_{M}
\end{array}\right]
$$


where the $i$-th column contains the samples $x_{i}(n)$,

$$
\mathbf{x}_{i}=\left[\begin{array}{c}
x_{i}(0) \\
\vdots \\
x_{i}(N-1)
\end{array}\right] .
$$

The time delays of the ensemble are contained in the vector $\boldsymbol{\theta}=\left[\begin{array}{lll}\theta_{1} & \cdots & \theta_{M}\end{array}\right]^{T}$.

In the present study, the time delay estimation problem is studied in terms of the correlation matrix $\mathbf{R}_{x}$. We will first show how the eigenvalues are related to the ML time delay estimator and the delay statistics. Then, guided by the results, we propose 80 an efficient implementation of the ML estimator, $\check{\boldsymbol{\theta}}_{\mathrm{ML}}$, and an alternative estimator, $\hat{\boldsymbol{\theta}}_{\mathrm{ER}}$, based on an eigenvalue ratio (ER), together with a one-step (OS) estimator, $\hat{\boldsymbol{\theta}}_{\mathrm{OS}}$, used for initialization of $\check{\boldsymbol{\theta}}_{\mathrm{ML}}$ and $\hat{\boldsymbol{\theta}}_{\mathrm{ER}}$.

We start by observing that for perfectly aligned signals, i.e., $x_{i}(n)=s(n)+v_{i}(n)$, the $N \times N$ intra-signal correlation matrix is given by

$$
\mathbf{R}_{x} \triangleq E\left[\mathbf{x}_{i} \mathbf{x}_{i}^{T}\right]=\mathbf{s s}^{T}+\sigma_{v}^{2} \mathbf{I}
$$

where $\mathbf{s}=\left[\begin{array}{lll}s(0) & \cdots & s(N-1)\end{array}\right]^{T}$ is easily shown to be proportional to the first eigenvector of $\mathbf{R}_{x}$. The eigenvalues are given by

$$
\lambda_{i}= \begin{cases}E_{s}+\sigma_{v}^{2}, & i=1 \\ \sigma_{v}^{2}, & i=2, \ldots, N\end{cases}
$$

85 where $E_{s}=\mathbf{s}^{T} \mathbf{s}$ is the signal energy. The eigenvector $\psi_{1}$ is proportional to $\mathbf{s}$, i.e, $\boldsymbol{\psi}_{1}=1 / \sqrt{E_{s}} \mathbf{s}$, whereas the remaining eigenvectors are chosen arbitrarily as long as they are orthogonal to $\psi_{1}$.

An estimate of $\mathbf{R}_{x}$ is obtained by

$$
\hat{\mathbf{R}}_{x}=\frac{1}{M} \mathbf{X X}^{T}
$$

When the ensemble is misaligned with small time delays $\theta_{i}$, an approximation of $90 \quad x_{i}(t)$ can be obtained by making use of the continuous-time counterpart to the model in $(1)$,

$$
x_{i}(t)=s\left(t-\theta_{i}\right)+v_{i}(t)
$$


For small $\theta_{i}$, the observed signal can be approximated by

$$
x_{i}(t) \approx s(t)-\theta_{i} s^{\prime}(t)+\frac{1}{2} \theta_{i}^{2} s^{\prime \prime}(t)+v_{i}(t),
$$

where $s^{\prime}(t)$ and $s^{\prime \prime}(t)$ denote the first and second derivative of $s(t)$, respectively. A second-order approximation of $x_{i}(t)$ is considered since the first-order terms will cancel when computing the expectations in $\mathbf{R}_{x}$, leaving only the second-order terms in the approximation of $\mathbf{R}_{x}$. For the second-order approximation of $\mathbf{R}_{x}$ to be complete, the terms resulting from the product of $s(t)$ with the second-order terms in $x_{i}(t)$ in (9) are also required, see below.

The intra-signal correlation matrix of the sampled counterpart of $x_{i}(t)$ in (9) can be expressed as

$$
\mathbf{R}_{x} \approx\left(\mathbf{s s}^{T}+\frac{\sigma_{\theta}^{2}}{2}\left(\mathbf{s s}^{\prime \prime T}+\mathbf{s}^{\prime \prime} \mathbf{s}^{T}\right)\right)+\sigma_{\theta}^{2} \mathbf{s}^{\prime} \mathbf{s}^{\prime T}+\sigma_{v}^{2} \mathbf{I}
$$

where $\mathbf{s}^{\prime}$ and $\mathbf{s}^{\prime \prime}$ are defined from $s^{\prime}(n)$ and $s^{\prime \prime}(n)$, respectively, in the same way as $\mathbf{s}$ is defined from $s(n)$. It can be shown that the eigenvalues of $\mathbf{R}_{x}$ are (see Appendix A)

$$
\lambda_{i} \approx \begin{cases}E_{s}-\sigma_{\theta}^{2} E_{s^{\prime}}+\sigma_{v}^{2}, & i=1 \\ \sigma_{\theta}^{2} E_{s^{\prime}}+\sigma_{v}^{2}, & i=2 \\ \sigma_{v}^{2}, & i=3, \ldots, N,\end{cases}
$$

where $E_{s^{\prime}}=\mathbf{s}^{\prime T} \mathbf{s}^{\prime}$. Then, recalling that $\sigma_{\theta}$ is the variance of the time delay, it is evident from (11) that maximization of $\lambda_{1}$ with respect to $\theta$ is equivalent to minimization of $\sigma_{\theta}^{2}$, thus reducing misalignment.

The eigenvectors $\boldsymbol{\psi}_{1}$ and $\boldsymbol{\psi}_{2}$ are approximately proportional to (see Appendix A)

$$
\begin{aligned}
& \psi_{1} \propto \mathbf{s}+\frac{\sigma_{\theta}^{2}}{2} \mathbf{s}^{\prime \prime}, \\
& \psi_{2} \propto \mathbf{s}^{\prime}
\end{aligned}
$$

For small $\theta_{i}$, and thus a small $\sigma_{\theta}^{2}, \boldsymbol{\psi}_{1}$ is approximately proportional to $\mathbf{s}$. The remaining eigenvectors can be chosen arbitrarily as long as they are orthogonal to $\psi_{1}$ and $\psi_{2}$.

With this formulation, $\mathbf{R}_{x}$ is characterized in terms of $\sigma_{\theta}$. Moreover, since $s\left(n-\theta_{i}\right)$ is always contained in $[0, N-1], E_{s}=\sum_{n=0}^{N-1} s_{i}^{2}\left(n-\theta_{i}\right)$ is independent of $\theta_{i}$ and

$$
\operatorname{tr}\left\{\mathbf{R}_{x}\right\}=\sum_{n=0}^{N-1} E\left[x_{i}^{2}(n)\right]=\sum_{n=0}^{N-1}\left(E\left[s_{i}^{2}\left(n-\theta_{i}\right)\right]+E\left[v_{i}^{2}(n)\right]\right)=E_{s}+N \sigma_{v}^{2}
$$


is invariant to $\theta_{i}$, emphasizing that $\lambda_{i}$ in (11) are approximate as their sum does not match the trace. Note also that $\lambda_{i}$ in (6) are exact, since no approximation was used to derive them.

\subsection{Maximum likelihood estimation}

This subsection shows that maximization of the most significant eigenvalue of the inter-signal sample correlation matrix is approximately the same as the well-known ML estimator of $\boldsymbol{\theta}$ [22]. This insight is essential for the development of a related estimator in Section 2.3. The ML estimator [22] is defined by

$$
\hat{\boldsymbol{\theta}}_{\mathrm{ML}}=\arg \max _{\boldsymbol{\theta}} \Lambda_{\triangle}(\boldsymbol{\theta})
$$

where the log-likelihood function $\Lambda_{\triangle}(\boldsymbol{\theta})$ equals (see Appendix B)

$$
\Lambda_{\triangle}(\boldsymbol{\theta})=\sum_{n} \sum_{i=1}^{M} \sum_{k>i}^{M} x_{k}\left(n+\theta_{k}\right) x_{i}\left(n+\theta_{i}\right) .
$$

Note that $\boldsymbol{\theta}$ in (14)-(15) denotes an optimization variable, not the delay parameter itself. Detailed analysis of this expression, together with the expression which defines the inter-signal sample correlation estimator [25], shows that $\Lambda_{\triangle}(\boldsymbol{\theta})$ is proportional to the sum of all elements of the upper triangular part of the $M \times M$ inter-signal sample correlation matrix.

$$
\hat{\mathbf{R}}_{x}^{\bullet}=\frac{1}{N} \mathbf{X}^{T} \mathbf{X} .
$$

Departing from this observation and from the second-order approximation of $x_{i}(t)$ in (9) we will show that maximization of the most significant eigenvalue of $\hat{\mathbf{R}}_{x}^{\bullet}$ is approximately the same as $\hat{\boldsymbol{\theta}}_{\mathrm{ML}}$. When sampling $x_{i}(t)$ and compiling all the observed samples at time $n$ in a vector, the $\mathrm{M}$ observations are compactly modeled by

$$
\mathbf{x}(n) \approx s(n) \mathbf{1}-s^{\prime}(n) \boldsymbol{\theta}+\frac{1}{2} s^{\prime \prime}(n) \boldsymbol{\theta}^{2}+\mathbf{v}(n),
$$

where

$$
\mathbf{x}(n)=\left[\begin{array}{c}
x_{1}(n) \\
\vdots \\
x_{M}(n)
\end{array}\right]
$$


$\mathbf{v}(n)$ defined analogously, $\boldsymbol{\theta}^{2}=\boldsymbol{\theta} \odot \boldsymbol{\theta}=\left[\begin{array}{lll}\theta_{1}^{2} & \cdots & \theta_{M}^{2}\end{array}\right]^{T}$, and $\mathbf{1}$ is the all-one $M \times 1$ vector. The related correlation matrix, determined by noting that the expectations are evaluated over " $n$ " rather than over " $i$ ", is given by

$$
\begin{aligned}
\mathbf{R}_{x}^{\bullet} & =E\left[\mathbf{x}(n) \mathbf{x}^{T}(n)\right] \\
& \approx \frac{1}{N}\left(E_{s} \mathbf{1 1}^{T}-\frac{E_{s^{\prime}}}{2}\left(\mathbf{1}^{2^{T}}+\boldsymbol{\theta}^{2} \mathbf{1}^{T}\right)\right)+\frac{E_{s^{\prime}}}{N} \boldsymbol{\theta} \boldsymbol{\theta}^{T}+\sigma_{v}^{2} \mathbf{I}
\end{aligned}
$$

where use is made of the fact that $E\left[\mathbf{1}^{T}\right]=\mathbf{0}_{\mathrm{M}, \mathrm{M}}$ and $E\left[\boldsymbol{\theta}^{2} \boldsymbol{\theta}^{T}\right]=\mathbf{0}_{\mathrm{M}, \mathrm{M}} ; \mathbf{0}_{\mathrm{M}, \mathrm{M}}$ denotes the $M \times M$ all-zero matrix. Use is also made of $E\left[s^{2}(n)\right]=\sum_{n=0}^{N-1} s^{2}(n) / N=$ $E_{s} / N$, and similarly $E\left[\left(s^{\prime}(n)\right)^{2}\right]=E_{s^{\prime}} / N, E\left[s(n) s^{\prime \prime}(n)\right]=E_{s s^{\prime \prime}} / N=-E_{s^{\prime}} / N$ and $E\left[s(n) s^{\prime}(n)\right]=E\left[s^{\prime \prime}(n) s^{\prime}(n)\right]=0$. Fourth order terms are discarded as already done before. The eigenvalues of $\mathbf{R}_{x}^{\bullet}$ are given by (see Appendix A)

$$
\lambda_{i}^{\bullet} \approx \begin{cases}\frac{E_{s} M}{N}-\frac{\sigma_{\theta}^{2} E_{s^{\prime}} M}{N}+\sigma_{v}^{2}, & i=1 \\ \frac{\sigma_{\theta}^{2} E_{s^{\prime}} M}{N}+\sigma_{v}^{2}, & i=2 \\ \sigma_{v}^{2}, & i=3, \ldots, M .\end{cases}
$$

The eigenvectors $\psi_{1}^{\bullet}$ and $\psi_{2}^{\bullet}$ are approximately proportional to (see Appendix A)

$$
\begin{aligned}
& \boldsymbol{\psi}_{1}^{\bullet} \propto \mathbf{1}-\frac{E_{s^{\prime}}}{2 E_{s}} \boldsymbol{\theta}^{2}, \\
& \boldsymbol{\psi}_{2}^{\bullet} \propto \boldsymbol{\theta} .
\end{aligned}
$$

Since the approximations in (9) imply that $\theta_{i}<<1$, and, consequently, $\theta_{i}^{2}<<1$, the eigenvector approximations in (21) can be further approximated by $\boldsymbol{\psi}_{1}^{\bullet} \approx 1 / \sqrt{M}$ and $\boldsymbol{\psi}_{2}^{\bullet} \approx \beta \boldsymbol{\theta}$, where $\beta$ is a proportionality factor.

Making use of the eigenvector equation, $\mathbf{R}_{x}^{\bullet} \psi_{i}^{\bullet}=\lambda_{i}^{\bullet} \psi_{i}^{\bullet}$, particularized for $i=1$, pre-multiplying both sides by $\psi_{1}^{\bullet T}$ and using the eigenvector approximation, we can write

$$
\mathbf{1}^{T} \mathbf{R}_{x}^{\bullet} \mathbf{1} \approx \lambda_{1}^{\bullet} M
$$

which leads to that $\lambda_{1}^{\bullet} M$ is approximately equal to the sum of all elements in $\mathbf{R}_{x}^{\bullet}$. Making use of the symmetry of $\hat{\mathbf{R}}_{x}^{\bullet}$, (22) becomes

$$
\mathbf{1}^{T} \hat{\mathbf{R}}_{x}^{\bullet} \mathbf{1}=\frac{2 \Lambda_{\triangle}(\mathbf{0})}{N}+\operatorname{tr}\left\{\hat{\mathbf{R}}_{x}^{\bullet}\right\} \approx \lambda_{1}^{\bullet} M
$$


where $\mathbf{0}$ is the all-zero $M \times 1$ vector. Analogous to earlier reasoning, $\operatorname{tr}\left\{\hat{\mathbf{R}}_{x}^{\bullet}\right\}=$ $E_{s} M / N+M \sigma_{v}^{2}$ is invariant to $\boldsymbol{\theta}$. Therefore, correcting the misaligned ensemble by a variable delay $\boldsymbol{\theta}$, as in (14), and maximizing $\Lambda_{\triangle}(\boldsymbol{\theta})$ with respect to $\boldsymbol{\theta}$, to obtain $\hat{\boldsymbol{\theta}}_{\mathrm{ML}}$, is approximately equal to the maximization of the $\lambda_{1}^{\bullet}(\boldsymbol{\theta})$, obtained from the alignedcorrected ensemble, so that the suboptimal ML estimator can be implemented by

$$
\check{\boldsymbol{\theta}}_{\mathrm{ML}} \triangleq \arg \max _{\boldsymbol{\theta}} \lambda_{1}^{\bullet}(\boldsymbol{\theta})
$$

where ${ }^{\prime \prime}$, denotes that the estimator is suboptimal, and $\lambda_{1}^{\bullet}(\mathbf{0})=\lambda_{1}^{\bullet}$ in (20). Note that the approximations to derive $\lambda_{1}^{\bullet}(\boldsymbol{\theta})$, i.e. (20) and (22), are now evaluated, not around the delays in the ensemble as in (9), but around the residual delays after alignment by the variable $\boldsymbol{\theta}$, and become more accurate the smaller these residual delays are, making the estimates in (14) and (24) equal at the position of the objective functions maximum. Analogously, $\sigma_{\theta}$ in (20), when associated with $\lambda_{1}^{\bullet}(\boldsymbol{\theta})$, is the variance of the residual delays. Since the maximum of $\lambda_{1}^{\bullet}(\boldsymbol{\theta})$ will always occur at $\boldsymbol{\theta}$ around the original delay, implying small residuals, the approximate expressions in (9), (21), and (22) remain largely accurate even for large delays, reinforcing the validity of $\check{\boldsymbol{\theta}}_{\mathrm{ML}}$ as surrogate of $\hat{\boldsymbol{\theta}}_{\mathrm{ML}}$.

The resulting estimates are determined up to a constant offset $\theta_{b}$, for all $\theta_{i}$. This results from the fact that an ensemble with signal cycles offset by $\theta_{b}$ while still preserving the compact support condition in $[0, N-1]$, will lead to the same eigenvectors $\lambda_{i}(\boldsymbol{\theta})$ and $\lambda_{i}^{\bullet}(\boldsymbol{\theta})$. The maximization of $\lambda_{i}^{\bullet}(\boldsymbol{\theta})$ yields estimates which are determined up to a constant since the maximum is not a point at the $M$ dimension delay space, but a hyperdiagonal line. This is easily proven by replacing $\theta_{i}$ in (8) by $\theta_{i}+\theta_{b}$, yielding

$$
x_{i}(t) \approx s\left(t-\theta_{b}\right)-\theta_{i} s^{\prime}\left(t-\theta_{b}\right)+\frac{1}{2} \theta_{i}^{2} s^{\prime \prime}\left(t-\theta_{b}\right)+v_{i}(t)
$$

which results in exactly the same eigenvalues, provided that the compact support condition is fulfilled. In practice, the delay offset is irrelevant since the interest is in the overall signal morphology irrespective of an offset. When the offset is relevant it can be easily corrected for by subtracting its mean. 


\subsection{Eigenvalue-based estimation}

By inspecting the eigenvalue structure in (20), it is evident that not only $\lambda_{1}^{\bullet}(\boldsymbol{\theta})$

165

The observations made above, for $\breve{\boldsymbol{\theta}}_{\mathrm{ML}}$, of time delay estimates offset, and approximations accuracy for large delays, also applies to $\hat{\boldsymbol{\theta}}_{\mathrm{ER}}$.

Although both $\Lambda^{\bullet}(\boldsymbol{\theta})$ and $\Lambda(\boldsymbol{\theta})$ result in the same estimator, they are related to different correlation matrices with dimensions $M \times M$ and $N \times N$, respectively. From an implementation viewpoint, the matrix with lower dimension is preferred. 


\subsection{One-step estimator}

The estimators $\check{\boldsymbol{\theta}}_{\mathrm{ML}}$ in (24) and $\hat{\boldsymbol{\theta}}_{\mathrm{ER}}$ in (28) both require computationally demanding maximization. Within the proposed eigenanalysis framework, a new one-step (OS) estimator is proposed. This estimator is suboptimal, but since it does not require maximization, it can be used for smart initialization of the maximization required in $\check{\boldsymbol{\theta}}_{\mathrm{ML}}$ and $\hat{\boldsymbol{\theta}}_{\mathrm{ER}}$. The OS estimator avoids maximization and can be derived by exploring the result that $\boldsymbol{\psi}_{2}^{\bullet}$ in (21) is approximately proportional to $\boldsymbol{\theta}$, i.e., $\boldsymbol{\psi}_{2}^{\bullet} \approx \beta \boldsymbol{\theta}$. The factor $\beta$ can be determined by making use of $\left\|\boldsymbol{\psi}_{2}^{\bullet}\right\|=1$ and $\boldsymbol{\theta}^{T} \boldsymbol{\theta}=M \sigma_{\theta}^{2}$, leading to that $\beta=1 / \sqrt{M \sigma_{\theta}^{2}}$. The OS estimator is defined by

$$
\begin{aligned}
\hat{\boldsymbol{\theta}}_{\mathrm{OS}} & \triangleq \frac{1}{\beta} \boldsymbol{\psi}_{2}^{\bullet}=\sqrt{M \sigma_{\theta}^{2}} \boldsymbol{\psi}_{2}^{\bullet} \\
& =\sqrt{\frac{\left(\lambda_{2}^{\bullet}-\sigma_{v}^{2}\right) N}{E_{s^{\prime}}}} \boldsymbol{\psi}_{2}^{\bullet},
\end{aligned}
$$

where the last equality comes from the introduction of $\lambda_{2}^{\bullet}$ in (20).

Before $\hat{\boldsymbol{\theta}}_{\mathrm{OS}}$ can be used, $\sigma_{v}^{2}$ and $E_{s^{\prime}}$ have to be determined-a problem whose solution depends on the application of interest. Since the noise is assumed to be stationary, making it possible to estimate $\sigma_{v}^{2}$ by the ensemble variance, it is computed in intervals where the signal energy is negligible [26].

An estimate for $E_{s^{\prime}}$ is obtained by first computing the ensemble average, then filtering to extract the main components of $s(n)$, and finally computing the energy $E_{s^{\prime}}$ from the differenced plus filtered signal. The sign uncertainty associated with $\boldsymbol{\psi}_{2}^{\bullet}$ can be solved by taking the sign that maximizes $\lambda_{1}^{\bullet}\left( \pm \hat{\boldsymbol{\theta}}_{\mathrm{os}}\right)$.

The OS estimator $\hat{\boldsymbol{\theta}}_{\text {oS }}$ can either be used separately, or to initialize the maximization of the ML and ER estimators, leading to a considerably reduced grid search.

\subsection{Maximization of objective functions}

Maximization of the two objective functions is performed using bound constrained particle swarm optimization [27, 28], implemented in the MATLAB function particleswarm (version 2015b), using a Toshiba laptop with an Intel Core i7-2640M processor. Figure 1 illustrates $\Lambda(\boldsymbol{\theta})$ for a small ensemble $(M=3)$ displayed for $\theta_{2}$ and $\theta_{3}$ at an SNR of $25 \mathrm{~dB}$, when $\theta_{1}$ is held fixed. 


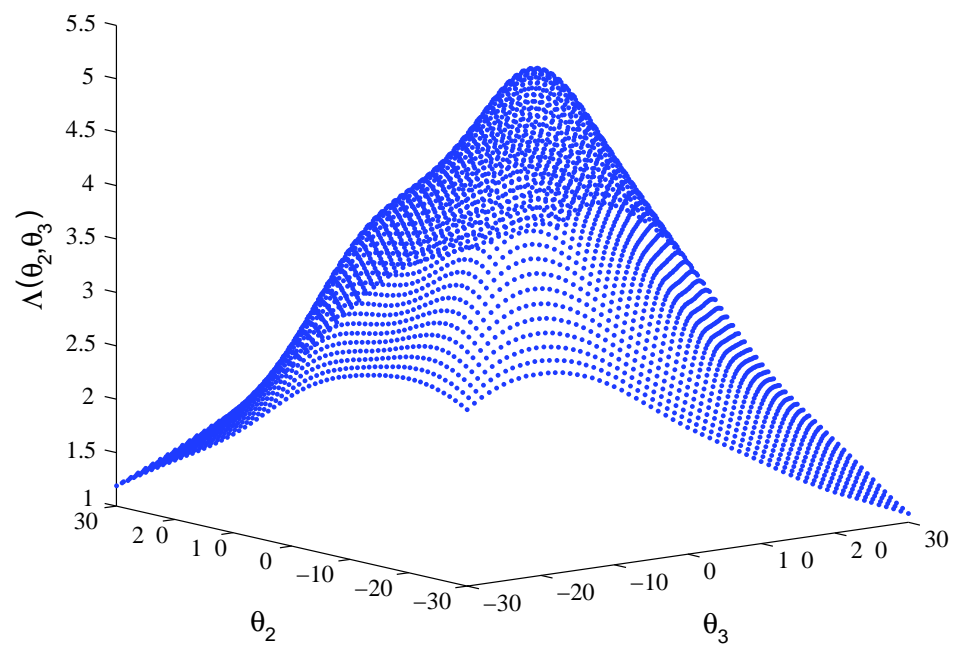

Figure 1: The objective function $\Lambda(\boldsymbol{\theta})$ displayed for $\theta_{2}$ and $\theta_{3}$ at an SNR of $25 \mathrm{~dB}$, when $\theta_{1}$ is held fixed. Its maximum occurs for $\left(\theta_{2}, \theta_{3}\right)=(0,0)$ which are identical to the true time delays of the simulation. The simulation model and the SNR are defined in Section 3.

\subsection{Amplitude and shape variability}

The signal model pursued in the present paper assumes that $s(n)$ has fixed amplitude and shape. However, this assumption may not be fulfilled, since, for example, the amplitude of heartbeats can vary considerably over time due to respiration. While the analysis of varying amplitude and shape on time delay estimation is outside the scope of the present paper, the implications of varying amplitude are briefly discussed in the following extended signal model:

$$
x_{i}(n)=a_{i} s\left(n-\theta_{i}\right)+v_{i}(n), \quad n=0, \ldots, N-1,
$$

where $a_{i}$ is a random amplitude with mean $m_{a}=1$ and variance $\sigma_{a}^{2}\left(\ll m_{a}^{2}\right)$. The variables $a_{i}$ and $\theta_{i}$ are assumed to be uncorrelated.

The eigenvalues of the correlation matrix for the model in (30) are given by

$$
\lambda_{i} \approx \begin{cases}\left(\sigma_{a}^{2}+1\right)\left(E_{s}-\sigma_{\theta}^{2} E_{s^{\prime}}\right)+\sigma_{v}^{2}, & i=1 \\ \left(\sigma_{a}^{2}+1\right) \sigma_{\theta}^{2} E_{s^{\prime}}+\sigma_{v}^{2}, & i=2 \\ \sigma_{v}^{2}, & i=3, \ldots, N\end{cases}
$$


and the corresponding eigenvalue ratio is

$$
\Lambda_{a}(\boldsymbol{\theta})=\frac{\lambda_{1}(\boldsymbol{\theta})}{\sum_{i=2}^{N} \lambda_{i}(\boldsymbol{\theta})} \approx \frac{E_{s}-\sigma_{\theta}^{2} E_{s^{\prime}}}{\sigma_{\theta}^{2} E_{s^{\prime}}+\frac{N \sigma_{v}^{2}}{\sigma_{a}^{2}+1}} .
$$

Analogously to (27), $\Lambda_{a}(\boldsymbol{\theta})$ is maximized when the signals with varying amplitudes are aligned.

Shape variability may also be present in the ensemble, showing up in $\lambda_{2}, \lambda_{3}$ and higher-order eigenvalues of (31) (all being in the denominator of $\Lambda_{a}(\boldsymbol{\theta})$ in (32)). Therefore, such variability does not influence the underlying design principle of the eigenvaluebased estimators. This observation assumes that the shape variability has lower energy than $s(n)$, being the case in most biomedical applications. Thus, the eigenvalue ratio in (27) should be well-suited for time delay estimation in the presence of shape variability.

\section{Simulation}

The present simulation results are based on a real respiratory flow signal from a patient with chronic heart failure (CHF) and periodic breathing, extracted from a database acquired with a pneumotachograph at a sampling rate of $250 \mathrm{~Hz}$ [29]. A representative respiratory flow cycle of about $2.5 \mathrm{~s}$ is extracted. Zero-valued samples are inserted symmetrically before and after the extracted cycle to produce a transient signal $s(n)$ extending over $6 \mathrm{~s}$, thereby allowing misalignments of up to 200 samples. Using this respiratory cycle, defining $s(n)$, we simulate ensembles of misaligned signals by repetitively delaying $s(n)$ to $s\left(n-\theta_{i}\right)$ and adding noise $v_{i}(n)$ to form the observed signal $x_{i}(n)$. The integer-valued time delay $\theta_{i}$ is uniformly distributed over the interval $[-\delta, \delta]$ implying a delay PDF with variance $\sigma_{\theta}^{2}=\delta^{2} / 3$. The signal-to-noise ratio (SNR) is defined as $10 \cdot \log \left(E_{s} / \sigma_{v}^{2}\right)$. An ensemble of 20 misaligned signals is shown in Fig. 2(b). Note that the selected respiratory flow cycle in Fig. 2(a) has similar peak flow and duration for inspiration and expiration, common in patients with chronic heart failure and periodic breathing [24]. This characteristic stands in contrast to normal subjects where peak flow and duration differ between inspiration and expiration.

The eigenvalue-based method involves only one parameter, namely, the maximum time shift $\Delta_{\max }$ defining the search interval $\left[-\Delta_{\max }, \Delta_{\max }\right]$ for finding the maximum 

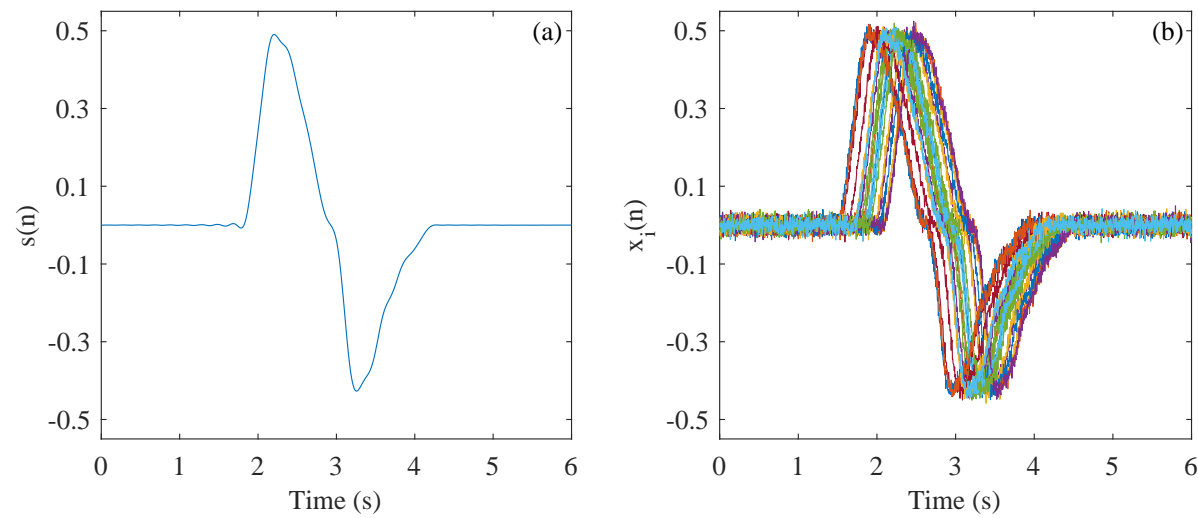

Figure 2: (a) A respiratory flow cycle and (b) a simulated misaligned ensemble, using $M=20$ and SNR $=25 \mathrm{~dB}$.

of the objective function. Here, $\Delta_{\max }=\delta$ guarantees that any introduced delay in the simulation can be optimally estimated in the grid search. $\delta$ is set to 80 samples, unless

\section{Real data}

The proposed estimator is also tested on a real data ensemble using a respiratory flow signal, recorded from a chronic heart failure patient with periodic breathing [24], 
sampled at $250 \mathrm{~Hz}$. The respiratory flow cycles are extracted from this signal, being different from the one used in the simulation. In these patients, abnormal evolution of the respiratory pattern (amplitude, morphology, etc) can trigger alarms on exacerbation of the underlying pathological process. For this purpose, respiratory cycle features such as amplitudes and slopes have been proposed for monitoring [24]. The features are computed from an ensemble average, $\hat{\mathbf{s}}$, to reduce the influence of noise. Also, time alignment prior to ensemble averaging is required to ensure that the low-pass filtering effect is minimized [2] when computing the average.

A signal ensemble from a patient composed of $M=20$ cycles is subject to averaging, before and after alignment. The segmentation of the cycles is determined by the zero-crossing at the onset of each respiratory flow cycle [24] up to the next onset of the subsequent cycle. The zero-crossings are determined from a low-pass filtered signal to reduce the influence of noise on the segmentation, minimizing instabilities around the zero-crossing location. To ensure that all cycles have the same length, they have been restricted to the shortest cycle length of the ensemble, here $3 \mathrm{~s}$. Assuming that the cycle-to-cycle variability in duration is relatively modest, it is reasonable to consider that the most part of the cycle is completely contained in the segmentation interval. The alignment is made by $\hat{\boldsymbol{\theta}}_{\mathrm{ER}}$ estimator, using $\delta=0.2 \mathrm{~s}$.

\section{Results}

The results presented in this section are computed using the algorithmic steps described below and in the pseudo code at Table 1. The performance is evaluated as described in point 3 .

1. Creation of the signal ensemble: from real or simulated signals.

2. Time delay estimation using $\check{\boldsymbol{\theta}}_{\mathrm{ML}}$ in (24), $\hat{\boldsymbol{\theta}}_{\mathrm{ER}}$ in (28), or $\hat{\boldsymbol{\theta}}_{\mathrm{OS}}$ in (29).

3. Computation of performance results which for simulated data is expressed in terms of the error metric $\sigma_{e}$ and for real data by presenting the ensemble average before and after alignment. 
Table 1: The pseudo code algorithm for obtaining the estimated delays $\hat{\boldsymbol{\theta}}$.

$\mathbf{X}=\left[\begin{array}{lll}\mathbf{x}_{1} & \cdots & \mathbf{x}_{M}\end{array}\right], \quad$ (matrix $\mathbf{X}$ creation)

$\hat{\mathbf{R}}_{x}^{\bullet}=\frac{1}{N} \mathbf{X}^{T} \mathbf{X}, \quad$ (autocorrelation matrix estimation, or $\hat{\mathbf{R}}_{x}=\frac{1}{M} \mathbf{X} \mathbf{X}^{T}$ if this is the preferred)

If one step initialization $\hat{\boldsymbol{\theta}}_{\mathrm{OS}}$

$\hat{\mathbf{R}}_{x}^{\bullet} \boldsymbol{\psi}_{2}^{\bullet}=\lambda_{2}^{\bullet} \boldsymbol{\psi}_{2}^{\bullet}, \quad$ (second eigenvector $\boldsymbol{\psi}_{2}^{\bullet}$ and eigenvalue $\lambda_{2}^{\bullet}$ estimation)

$\hat{\boldsymbol{\theta}}_{\mathrm{OS}}=\sqrt{\frac{\left(\lambda_{2}^{\bullet}-\sigma_{v}^{2}\right) N}{E_{s^{\prime}}}} \boldsymbol{\psi}_{2}^{\bullet}, \quad$ (one step delay $\hat{\boldsymbol{\theta}}_{\text {os }}$ estimation)

$x_{i}(n) \leftarrow x_{i}\left(n+\hat{\theta}_{i, \mathrm{os}}\right), \quad i=1, \ldots, M \quad$ (signal ensemble delay correction)

$\mathbf{X}=\left[\mathbf{x}_{1} \cdots \mathbf{x}_{M}\right], \quad\left(\hat{\boldsymbol{\theta}}_{\mathrm{OS}}\right.$ delay corrected initialized ensemble matrix $\mathbf{X}$ construction $)$

end

If $\check{\boldsymbol{\theta}}_{\mathrm{ML}}$ or $\hat{\boldsymbol{\theta}}_{\mathrm{ER}}$ estimate

for $\boldsymbol{\theta} \in$ "grid search" (grid required by the maximization rule, in this case particle swarm) $x_{i}\left(\theta_{i}, n\right) \leftarrow x_{i}\left(n+\theta_{i}\right), \quad i=1, \ldots, M \quad$ (signal ensemble delay correction)

$\mathbf{X}(\boldsymbol{\theta})=\left[\mathbf{x}_{1}(\boldsymbol{\theta}) \cdots \mathbf{x}_{M}(\boldsymbol{\theta})\right], \quad$ (ensemble matrix $\mathbf{X}$ reconstruction)

$\hat{\mathbf{R}}_{x}^{\bullet}(\boldsymbol{\theta})=\frac{1}{N} \mathbf{X}^{T}(\boldsymbol{\theta}) \mathbf{X}(\boldsymbol{\theta}) \quad$ (autocorrelation matrix estimation)

$\hat{\mathbf{R}}_{x}^{\bullet}(\boldsymbol{\theta}) \boldsymbol{\psi}_{i}^{\bullet}=\lambda_{i}^{\bullet}(\boldsymbol{\theta}) \boldsymbol{\psi}_{i}^{\bullet} \quad i=1, \ldots, M \quad$ (eigenvalue $\lambda_{i}^{\bullet}(\boldsymbol{\theta})$ estimation)

$\Lambda^{\bullet}(\boldsymbol{\theta})=\lambda_{i}^{\bullet}(\boldsymbol{\theta}) / \sum_{i=2}^{M} \lambda_{i}^{\bullet}(\boldsymbol{\theta}) \quad$ (objective function estimation)

end

If $\check{\boldsymbol{\theta}}_{\mathrm{ML}}$ estimate

$\check{\boldsymbol{\theta}}_{\mathrm{ML}}=\arg \max _{\boldsymbol{\theta} \in \text { grid search }} \lambda_{1}^{\bullet}(\boldsymbol{\theta}) \quad\left(\check{\boldsymbol{\theta}}_{\mathrm{ML}}\right.$ estimation by maximization with particle swarm $)$

end

If $\hat{\boldsymbol{\theta}}_{\mathrm{ER}}$ estimate

$\hat{\boldsymbol{\theta}}_{\mathrm{ER}}=\arg \max _{\boldsymbol{\theta} \in \text { grid search }} \Lambda^{\bullet}(\boldsymbol{\theta}) \quad\left(\hat{\boldsymbol{\theta}}_{\mathrm{ER}}\right.$ estimation by maximization with particle swarm $)$

end

end

$\hat{\boldsymbol{\theta}} \in\left\{\hat{\boldsymbol{\theta}}_{\mathrm{OS}}, \hat{\boldsymbol{\theta}}_{\mathrm{ER}}\right.$ or $\left.\check{\boldsymbol{\theta}}_{\mathrm{ML}}\right\}$ (final delay estimate). 

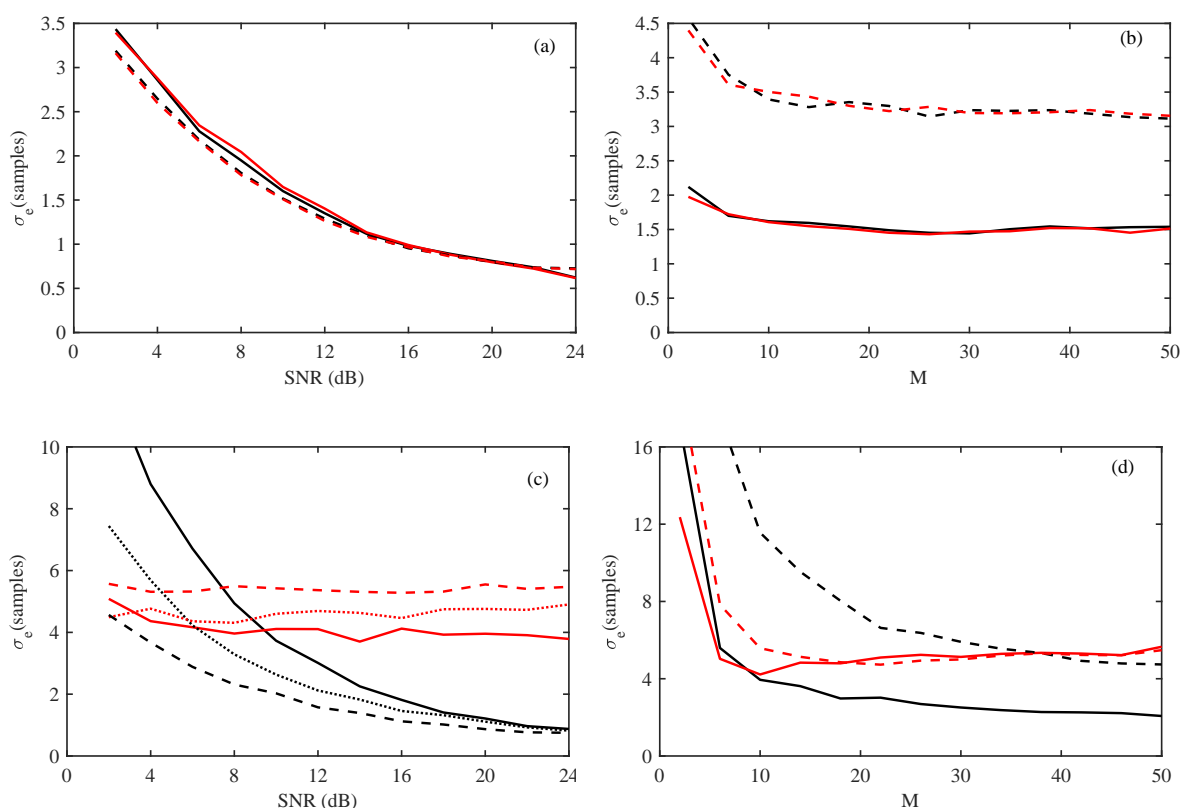

Figure 3: The time delay estimation error $\sigma_{e}$, computed, as a function of: (a) SNR for $M=10$ (solid line), and $M=50$ (dashed line), using $\hat{\boldsymbol{\theta}}_{\mathrm{ER}}$ (black line) and $\check{\boldsymbol{\theta}}_{\mathrm{ML}}$ (red line); (b) $M$ for SNR $=10 \mathrm{~dB}$ (solid line), $\mathrm{SNR}=2 \mathrm{~dB}$ (dashed line), $\delta=10$, using $\hat{\boldsymbol{\theta}}_{\mathrm{ER}}$ (black line) and $\check{\boldsymbol{\theta}}_{\mathrm{ML}}$ (red line); (c) SNR in $\mathrm{dB}$ for $M=10$ (solid line), $M=20$, (dotted line), and $M=50$ (dashed line), $\delta=80$, using $\hat{\boldsymbol{\theta}}_{\mathrm{W}}$ (black line) and $\hat{\boldsymbol{\theta}}_{\mathrm{os}}$ (red line); (d) $M$ for SNR $=10 \mathrm{~dB}$ (solid line), SNR $=2 \mathrm{~dB}$ (dotted line), $\delta=80$, using $\hat{\boldsymbol{\theta}}_{\mathrm{W}}$ (black line), and $\hat{\boldsymbol{\theta}}_{\mathrm{OS}}$ (red line).

\subsection{Performance of the ER and ML estimators}

Figure 3(a) shows that the two estimators have similar performance in terms of $\sigma_{e}$ for different SNRs, both deteriorating as the SNR decreases. Larger ensembles are associated with better performance, particularly at low SNR. Figure 3(b) presents $\sigma_{e}$ as a function of $M$ for SNR $=2$ and $10 \mathrm{~dB}$, showing that $\sigma_{e}$ is largely independent of $\mathrm{M}$, except for small size of the ensemble and low SNR.

The objective functions $\Lambda\left(\hat{\boldsymbol{\theta}}_{\mathrm{ER}}\right)$ and $\lambda_{1}\left(\check{\boldsymbol{\theta}}_{\mathrm{ML}}\right)$, corresponding to optimally aligned ensembles, are shown in Fig. 4 as a function of SNR. The log-likelihood (objective) function of the ML estimator decreases as the SNR increases since $\sigma_{v}^{2}$ in $\lambda_{i}\left(\check{\boldsymbol{\theta}}_{\mathrm{ML}}\right)$ is additive. On the other hand, $\Lambda\left(\hat{\boldsymbol{\theta}}_{\mathrm{ER}}\right)$ behaves in the opposite way since it increases as the SNR increases. This behavior is explained by the fact that $\Lambda\left(\hat{\boldsymbol{\theta}}_{\mathrm{ER}}\right)$ involves the term $(N-1) \sigma_{v}^{2}$ in the denominator, thereby resulting in an inverse relation to the noise that dominates over $\sigma_{v}^{2}$ in the numerator, cf. (20). 


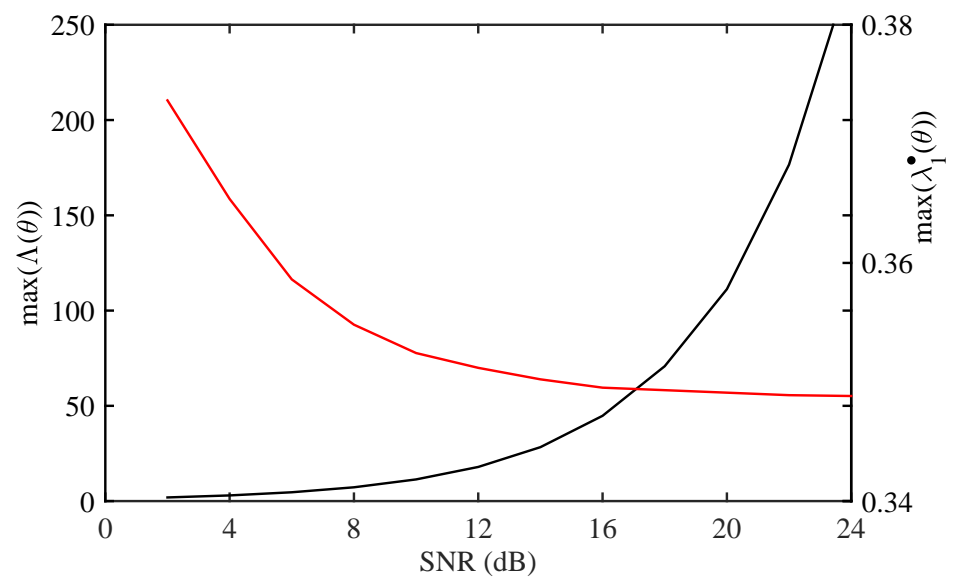

Figure 4: The objective functions $\Lambda\left(\hat{\boldsymbol{\theta}}_{\mathrm{ER}}\right)$ (black line) and $\lambda_{1}^{\bullet}\left(\check{\boldsymbol{\theta}}_{\mathrm{ML}}\right)$ (red line) as a function of SNR for optimally aligned ensembles and $M=10$.

\subsection{Performance of the OS estimator}

The performance of $\hat{\boldsymbol{\theta}}_{\text {os }}$ estimator is presented when used separately. From these results it can be evaluated the potential of this estimator to work either separately or in combination with the maximization estimator. The range of the reduction in the grid search size, when initialized by $\hat{\boldsymbol{\theta}}_{\text {os }}$, can be inferred by evaluating the residual error of $\hat{\boldsymbol{\theta}}_{\text {os }}$, which will become the minimum required grid search of the estimators involving maximization. The error $\sigma_{e}$ of $\hat{\boldsymbol{\theta}}_{\mathrm{oS}}$ is presented as a function of SNR in Fig. 3(c), for different $M$. For comparison, $\sigma_{e}$ of the Woody method $\hat{\boldsymbol{\theta}}_{\mathrm{w}}$ is included [16]. It is obvious that the performance of $\hat{\boldsymbol{\theta}}_{\mathrm{OS}}$ is almost independent of the SNR, with better performance for smaller $M$. On the contrary, $\hat{\boldsymbol{\theta}}_{\mathrm{w}}$ performs less well for smaller ensemble sizes since the required, intermediate ensemble average is then noisier. Another observation from this figure is that $\sigma_{e}$ increases for $\hat{\boldsymbol{\theta}}_{\mathrm{w}}$ as the SNR decreases, again explained by an increasingly noisy intermediate ensemble average. For $M=50, \sigma_{e}$ of $\hat{\boldsymbol{\theta}}_{\mathrm{w}}$ is very close to that of $\check{\boldsymbol{\theta}}_{\mathrm{ML}}$ in Fig. 3(a), demonstrating that the improvement achieved with $\check{\boldsymbol{\theta}}_{\mathrm{ML}}$ becomes more pronounced for smaller $M$ [22].

For low SNRs and small $M, \hat{\boldsymbol{\theta}}_{\text {os }}$ performs better than $\hat{\boldsymbol{\theta}}_{\mathrm{w}}$, see Fig. 3(c). This result, combined with the result that the performance of $\hat{\boldsymbol{\theta}}_{\mathrm{os}}$ is almost independent of the SNR and the property that $\hat{\boldsymbol{\theta}}_{\text {os }}$ is a one-step estimator, makes $\hat{\boldsymbol{\theta}}_{\text {os }}$ a better candidate for initialization of the maximization required in the ER and ML estimators. By comparing 
the results in Fig. 3(c) with those in Figs. 3(a) and 3(b) for SNR $=2$ and 10 dB, we note that the performance of $\hat{\boldsymbol{\theta}}_{\mathrm{OS}}$ and $\hat{\boldsymbol{\theta}}_{\mathrm{W}}$, as expected, is always lower than that of the $\check{\boldsymbol{\theta}}_{\mathrm{ML}}$ or $\hat{\boldsymbol{\theta}}_{\mathrm{ER}}$.

Figure 3(d) shows $\sigma_{e}$ as a function of $M$ for $\mathrm{SNR}=2$ and $10 \mathrm{~dB}$. The result in Fig. 3(c), showing that $\hat{\boldsymbol{\theta}}_{\mathrm{w}}$ performs less well for smaller $M$ whereas the reverse occurs for $\hat{\boldsymbol{\theta}}_{\mathrm{OS}}$, is again demonstrated since the performance of $\hat{\boldsymbol{\theta}}_{\mathrm{OS}}$ deteriorates as $M$ increases, while the performance of $\hat{\boldsymbol{\theta}}_{\mathrm{w}}$ improves as $M$ increases. From Fig. 3(d) it is observed that this reverted behaviour, favoring $\hat{\boldsymbol{\theta}}_{\mathrm{OS}}$ over $\hat{\boldsymbol{\theta}}_{\mathrm{W}}$ for low $M$ values, remains valid for larger $M$ range the lower SNR becomes; up to $M=10$ for SNR $=10 \mathrm{~dB}$ and up to $M=40$ for $\mathrm{SNR}=2 \mathrm{~dB}$.

Figure 5(a) presents the performance of $\hat{\boldsymbol{\theta}}_{\text {os }}$ as a function of SNR for different $\delta$. This result has particular relevance since it quantifies the impact of $\delta$ on the approximations associated with (9) and (19). From Fig. 5(a), $\sigma_{e}$ reduces, as expected, since $\delta$ becomes increasingly smaller. For a small $\delta$ (i.e., 10 or 15) and SNRs below $10 \mathrm{~dB}$, however, $\sigma_{e}$ increases as $\delta$ decreases_-a result which may be ascribed to the competing effects between time delays and noise in $\hat{\boldsymbol{\theta}}_{\mathrm{OS}}$, cf. (29). Figure 5(b) shows $\sigma_{e}$ for $\hat{\boldsymbol{\theta}}_{\mathrm{OS}}$ as well as for $\hat{\boldsymbol{\theta}}_{\mathrm{ER}}$ and $\check{\boldsymbol{\theta}}_{\mathrm{ML}}$ as a function of $\delta$, demonstrating that the performance of the latter two estimators are independent of $\delta$.

\subsection{Computational load}

Figure 5(a) demonstrates that initialization based on $\hat{\boldsymbol{\theta}}_{\text {os }}$ for the maximization required in $\hat{\boldsymbol{\theta}}_{\mathrm{ER}}$ and $\check{\boldsymbol{\theta}}_{\mathrm{ML}}$ implies that the original grid search can be constrained. For the most unfavorable case, when inspecting $\sigma_{e}$ for large time delays, i.e., $\delta=100$, it is obvious that the remaining estimation error is less than 10 samples. However, the remaining misalignment is to be handled by the maximization-based estimators. By using $\hat{\boldsymbol{\theta}}_{\mathrm{os}}$ for initialization, the grid search can be constrained to a conservative value larger than $2 \sigma_{e}$, resulting in about 20 samples, which, in turn, translates to a remarkably smaller grid. Using $\delta=100$ to estimate the reduced grid size, the brute force search leads to a reduction factor in computation time of $\delta^{M} /(\delta / 5)^{M}=5^{M}$. The dependence of $\sigma_{e}$ on $\delta$, obvious from Fig. 5(a), is a consequence of the fact that the larger the delay is in the model in (9) the less accurate is the approximation in $\hat{\boldsymbol{\theta}}_{\mathrm{os}}$, and therefore its 

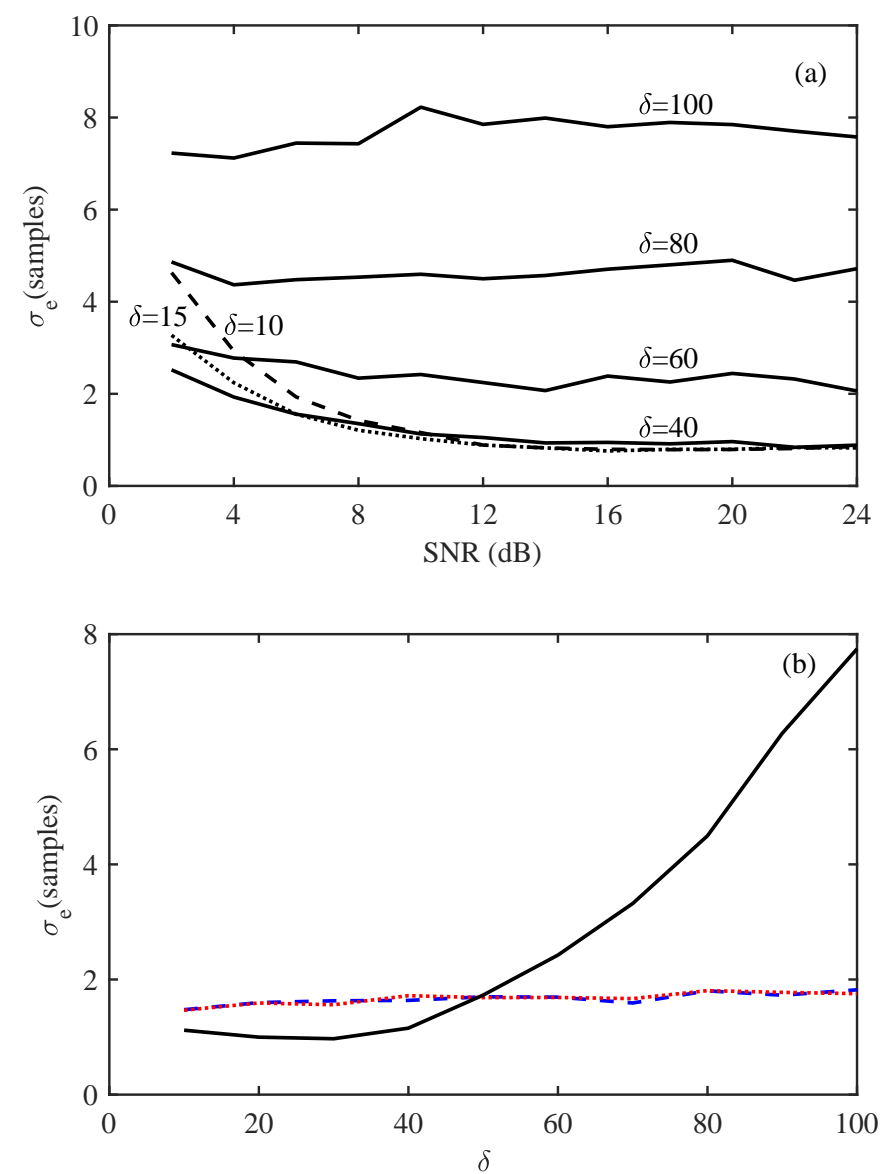

Figure 5: (a) The time delay estimation error $\sigma_{e}$ as a function of SNR for $M=20, R=50$, using $\hat{\boldsymbol{\theta}}_{\text {OS }}$ for different values of $\delta$ as indicated on the plot, $\delta \in\{40,60,80,100\}$ (solid lines), $\delta=15$ (dotted line), and $\delta=10$ (dashed line). (b) The time delay estimation error $\sigma_{e}$ as a function of $\delta$ for $M=20$ and SNR $=10 \mathrm{~dB}$, using $\hat{\boldsymbol{\theta}}_{\mathrm{ER}}$ (dashed blue line), $\check{\boldsymbol{\theta}}_{\mathrm{ML}}$ (dotted red line), and $\hat{\boldsymbol{\theta}}_{\mathrm{OS}}$ (black solid line).

performance, evaluated by $\sigma_{e}$, deteriorates.

Using instead particle swarm optimization, the saving factor has to be estimated experimentally. Figure 6(a) presents the average computation time for $\hat{\boldsymbol{\theta}}_{\mathrm{ER}}$ and $\check{\boldsymbol{\theta}}_{\mathrm{ML}}$ as a function of $M$ for different $\delta$, averaged over SNRs ranging from 2 to $24 \mathrm{~dB}$. Comparing the results in Figure 6(a) for different $\delta$, it is obvious that the saving factor is much smaller than that of brute force maximization. The factor may be estimated by comparing the computation time for $\delta=100$ and $\delta=10$ for $M=50$, leading to a saving factor of approximately 1.5 . For smaller $M$, the saving factor decreases and

becomes increasingly insignificant. 

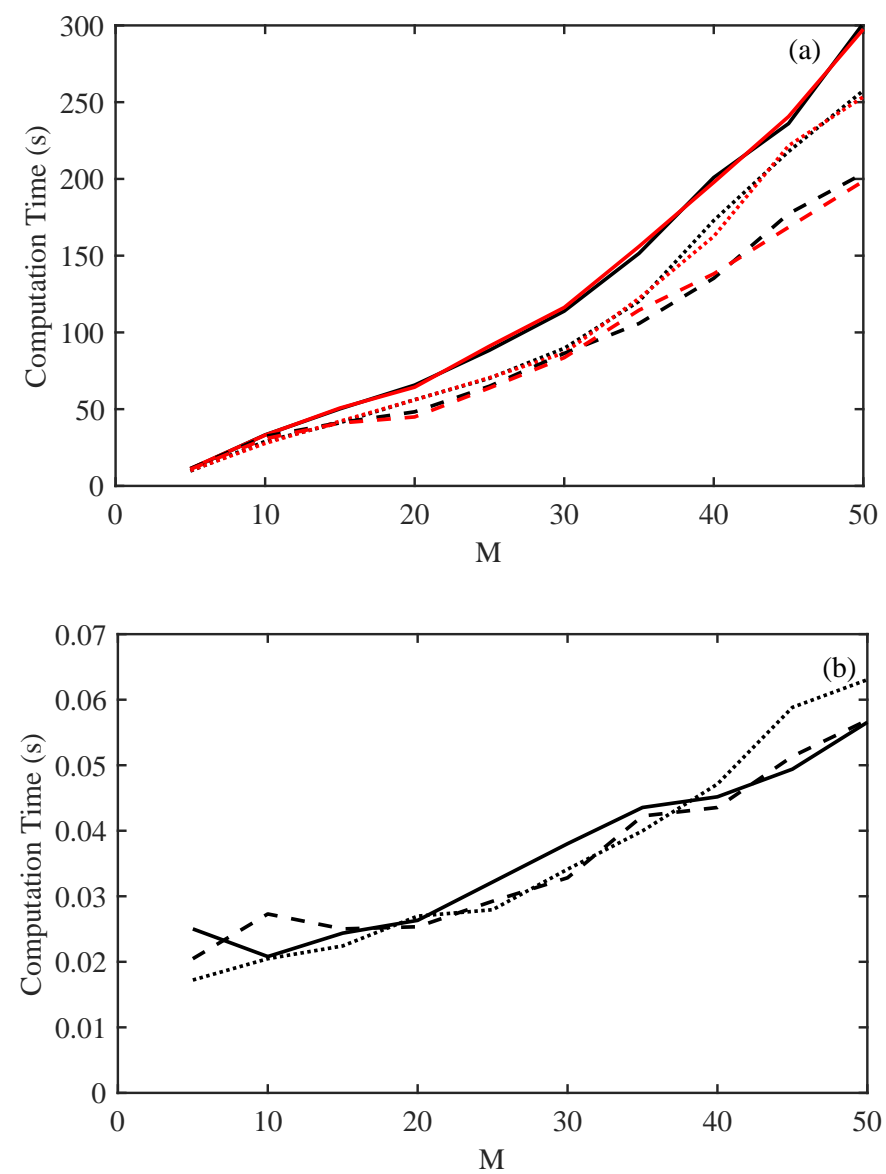

Figure 6: The computation time as a function of $M$, with $\delta$ set to 10 (dashed line), 20 (dotted line), and 100 (solid line), $R=50$, using (a) $\hat{\boldsymbol{\theta}}_{\mathrm{ER}}$ (black line) and $\check{\boldsymbol{\theta}}_{\mathrm{ML}}$ (red line), and (b) $\hat{\boldsymbol{\theta}}_{\mathrm{OS}}$ (black line). The vertical scales of (a) and (b) differ as a consequence of the maximization required in the ER-based methods.

Figure 6(b) presents the computation time for $\hat{\boldsymbol{\theta}}_{\mathrm{os}}$ as a function of $M$, being drastically faster than those of $\hat{\boldsymbol{\theta}}_{\mathrm{ER}}$ and $\check{\boldsymbol{\theta}}_{\mathrm{ML}}$ since no maximization is performed. As expected, the computation time increases with $M$. The computation time of the $\hat{\boldsymbol{\theta}}_{\mathrm{w}}$ has been omitted since it is not relevant in the present context.

\subsection{Real data results}

The patient data ensemble, described in Sec. 4, is presented in Fig. 7(a). In the ensemble, each respiratory cycle has, in addition to different time delay and noise, variability in shape, suggesting that the model in (30) involving amplitude variability, is more adequate. With certain shape variability, the proposed estimators will still work 
smaller values of $\mathrm{M}$ in the averaging, at the cost of less noise reduction.
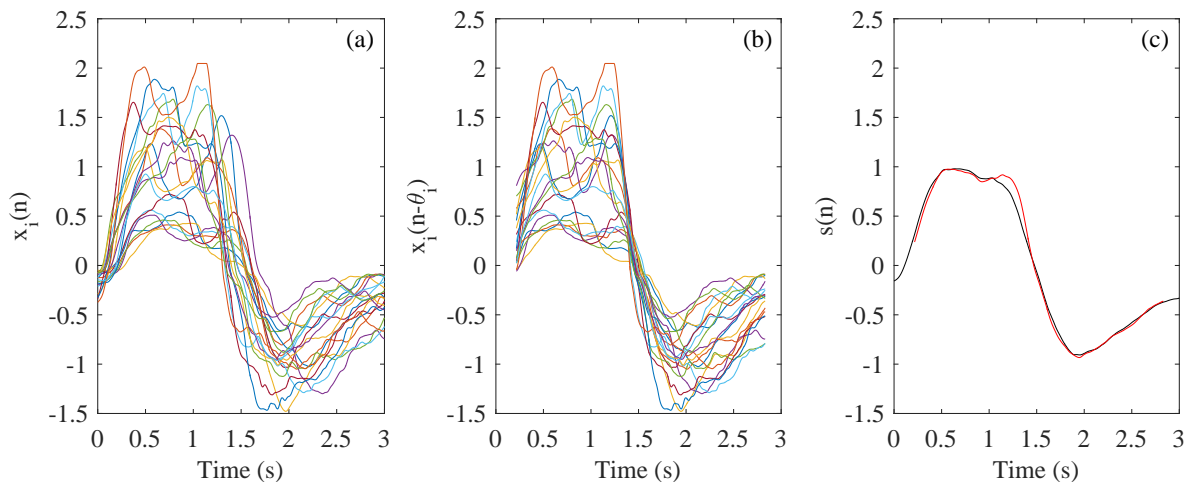

Figure 7: Ensemble with real respiratory flow cycles. (a) Ensemble extracted from signal segmentation, (b) ensemble after time alignment using $\hat{\boldsymbol{\theta}}_{\mathrm{ER}}$, and (c) the respiratory cycle, $\hat{\mathbf{s}}$, estimated by averaging the original ensemble (black), and the aligned ensemble (red).

\section{Discussion}

\section{Eigenvalue-based estimator}

The present paper proposes two time delay estimators based on eigenanalysis, embracing either maximization of an eigenvalue ratio $\left(\hat{\boldsymbol{\theta}}_{\mathrm{ER}}\right)$ or maximization of the first eigenvalue $\left(\check{\boldsymbol{\theta}}_{\mathrm{ML}}\right)$. The estimators have identical performance. Of these two estimators, $\check{\boldsymbol{\theta}}_{\mathrm{ML}}$ is the simpler one to implement, although no significant difference exist between 
the two with respect to computation time. Inspection of the approximations in (26)(27) suggests that maximization of the numerator together with minimization of the denominator, as in $\hat{\boldsymbol{\theta}}_{\mathrm{ER}}$, would yield better performance than would maximization of the numerator only, as in $\check{\boldsymbol{\theta}}_{\mathrm{ML}}$. However, recalling that $\operatorname{tr}\left\{\mathbf{R}_{x}\right\}$ and $\operatorname{tr}\left\{\mathbf{R}_{x}^{\bullet}\right\}$ are invariant to time delays, it is noted that the denominator in (26) equals $\operatorname{tr}\left\{\mathbf{R}_{x}^{\bullet}\right\}-\lambda_{i}^{\bullet}$ which implies that maximization of the numerator and minimization of the denominator are exactly the same, thus justifying the obtained results on identical performance of $\hat{\boldsymbol{\theta}}_{\mathrm{ER}}$ and $\check{\boldsymbol{\theta}}_{\mathrm{ML}}$. The objective functions $\Lambda\left(\hat{\boldsymbol{\theta}}_{\mathrm{ER}}\right)$ and $\lambda_{1}^{\bullet}\left(\check{\boldsymbol{\theta}}_{\mathrm{ML}}\right)$ have a reverted dependence with SNR for optimally aligned ensembles, see Fig. 4, as justified from inspection of (20) and (26).

For large $\delta$, Fig. 5(b) shows that the performance of $\hat{\boldsymbol{\theta}}_{\mathrm{ER}}$ and $\check{\boldsymbol{\theta}}_{\mathrm{ML}}$ do not deteriorate, although the expressions in (26) and (27) become less accurate as $\theta$ becomes larger. This result was already justified when introducing $\check{\boldsymbol{\theta}}_{\mathrm{ML}}$ in (24) and same conclusions can be reach analyzing the fact that both estimators reduce to maximization of $\lambda_{1}$. The first eigenvector of the correlation matrix may be regarded as the vector generating the first principal component of the signal ensemble [25], where the corresponding eigenvalue $\lambda_{1}$ is known to increase when the morphological variability of the ensemble decreases. In the model in (8), the ensemble variability is given by the noise variance, being invariant to time delays when the noise is stationary, plus the signal variance, reducing to zero for perfect alignment. Thus, this observation justifies that the maximization of $\lambda_{1}$ always results in an optimal estimator irrespective of the degree of time delay dispersion. If higher-order approximation terms in (26) and (27) had been considered to handle large $\theta_{i}$, the resulting expression would have become much more complicated and more difficult to interpret. However, the previous observation shows that the maximization of the resulting expression will still result in an optimal estimator.

Eigenvalue-based estimation, based on either $\hat{\boldsymbol{\theta}}_{\mathrm{ER}}$ or $\check{\boldsymbol{\theta}}_{\mathrm{ML}}$, represents an alternative way of implementing the ML estimator, cf. (23) and Fig. 3. These two estimators may benefit from efficient implementations of algorithms for eigenvalue decomposition, avoiding the triple summation in (15) and the need for an intermediate estimate of $s(n)$ [22]. Fig. 3(b) shows that performance gets better as $M$ increases for low of about $0 \mathrm{~dB}$ SNR as a result of better "learning" of the underlaying signal shape $s(n)$, 
whereas this learning is negligible at an SNR of about $10 \mathrm{~dB}$ or higher. distribution including a offset will result in a delayed estimate of $s(n)$, typically irrelevant in biomedical signal analysis where the information is on the overall shape, 
as already discussed when introducing the maximization estimators. In situations like

the evoked trigger is relevant, this offset can be easily corrected by subtracting the estimates mean. Concerning the stationarity assumption (\#3), it is reasonable to assume that signals recorded during resting conditions have fixed noise variance. Signals with large artifacts and intermittent disturbances are typically excluded before time alignment, making an assumption of a time-varying noise variance unnecessary.

One-step estimator

By analyzing the eigenvector structure of the approximate inter-signal correlation matrix $\mathbf{R}_{x}^{\bullet}$ in (19) for small time delays, the proposed one-step estimator $\hat{\boldsymbol{\theta}}_{\mathrm{Os}}$, being proportional to $\psi_{2}^{\bullet}$, outperforms the Woody method for low SNRs and small $M$, but not for high SNRs and large $M$, see Fig. 3. This $M$ value can reach up to 40 cycles, see Fig. 3(d), when the SNR approaches $0 \mathrm{~dB}$. The one-step estimator is of particular interest for initialization of the maximization-based estimators, leading to a reduction in computation time. This type of initialization is suitable to use in devices with constraints on power consumption, e.g., implantable devices, but less so in off-line applications. The use of $\hat{\boldsymbol{\theta}}_{\mathrm{OS}}$ is attractive since it draws on the framework of the inter-signal correlation matrix and eigenanalysis.

Since the computational saving factor is likely to depend on $s(n)$, a "learning step" will be required to determine the extent with which the grid search should be constrained. The learning step should take its starting point from results analogous to those in Fig. 5(a). Also, an estimate of $s(n)$ is required, e.g., obtained by ensemble averaging.

The computation of $\hat{\boldsymbol{\theta}}_{\mathrm{OS}}$ requires estimations of $\sigma_{v}^{2}$ and $E_{s^{\prime}}$ as described in Section 2.4. Alternative methods to estimating $\sigma_{v}^{2}$ is to use the higher-order eigenvalues, i.e, $\hat{\sigma}_{v}^{2}=\sum_{i=i_{0}}^{N} \lambda_{i} /\left(N-i_{0}+1\right)$, where $i_{0}$ is chosen such that signal shape variability 465 is avoided. For $E_{s^{\prime}}$, an alternatively strategy can be obtained by observing that $\mathbf{s}$ is essentially proportional to the first eigenvector of $\mathbf{R}_{x}$ in (12) so that an estimate of $E_{s^{\prime}}$ is obtained from $\hat{E}_{s^{\prime}} \approx\left(\lambda_{1}-\sigma_{v}^{2}\right) E_{\psi_{1}^{\prime}}$.

From inspection of Fig. 5(a) a iterative estimation process can be suggested by 
recurrent application of $\hat{\boldsymbol{\theta}}_{\mathrm{os}}$, particularly for large SNR,leading to better estimates.

\section{Implications of misalignment}

It is well-known that increased time delay jitter attenuates higher frequencies of the ensemble average [2]. Assuming an ensemble of $M=10$ signals, an SNR of $15 \mathrm{~dB}$, and a sampling rate of $1000 \mathrm{~Hz}$, the $3 \mathrm{~dB}$ cut-off frequency caused by $\sigma_{e}$ is approximately $150 \mathrm{~Hz}$ for eigenvalue-based alignment. For the OS estimator, the cut-off frequency drops to approximately below $90 \mathrm{~Hz}$. Such a substantial drop in cut-off frequency has repercussions in applications such as high-frequency ECG analysis, where an ensemble of QRS complexes is averaged and bandpass filtered (150$250 \mathrm{~Hz}$ ) [30, 31]; thus, an SNR higher than $15 \mathrm{~dB}$ should be employed. Better performance of $\hat{\boldsymbol{\theta}}_{\mathrm{ER}}$ not only has implications on ensemble averaging, but even more so on the estimation of ensemble variance where better accuracy is required of the time delay estimates [26].

The real data example presented in Fig. 7(c) illustrates the effects on the amplitude and the slope of a respiratory cycle, both more pronounced after alignment. These changes are the result of the increase in the cut-off frequency introduced by averaging after alignment [2]. This example is descriptive in nature, and does not pretend to be a clinical validation which is outside the scope of this study.

\section{Maximization}

In the present paper, particle swarm optimization [32] has been used, while other techniques were not investigated. Therefore, it may be possible that other techniques may offer faster convergence or come with less computational cost. The computation time has been analyzed in relative terms since the computation time in absolute terms are platform-dependent. While the maximization here presented is restricted to integer values, if required, a finer temporal resolution than that provided by the sampling rate can be obtained by interpolation. Once the optimal value is reached, a grid can be easily computed around this value. 


\section{Conclusions}

The present study introduces and evaluates novel methods for time delay estimation based on the eigenvalues of the sample correlation matrix of the signal ensemble. It is shown that the ML estimator can be implemented by maximizing either the first eigenvalue of this matrix, or, equivalently, a ratio defined by its eigenvalues. A one-step estimator is proposed based on the second eigenvector of the inter-signal correlation matrix. When using the one-step estimator for initialization, a reduction in computation time of the estimators involving maximization can be achieved.

\section{Appendix A}

This appendix derives $\lambda_{1}$ for a second-order approximation of $\mathbf{R}_{x}$, see (10). First, we observe that $s(t)$ and $s^{\prime}(t)$ are orthogonal, i.e.,

$$
\begin{aligned}
\int_{-\infty}^{\infty} s(t) s^{\prime}(t) d t & =\frac{1}{2 \pi} \int_{-\infty}^{\infty} S(\Omega)(-\jmath \Omega) S^{*}(\Omega) d \Omega \\
& =-\frac{1}{2 \pi} \int_{-\infty}^{\infty} \jmath \Omega|S(\Omega)|^{2} d \Omega=0 .
\end{aligned}
$$

With the same argument, $s^{\prime}(t)$ and $s^{\prime \prime}(t)$ are also orthogonal. The cross-energy between the signal $s(t)$ and its second derivative $s^{\prime \prime}(t)$ is always negative, and equal to minus the energy of the derivative, since

$$
\begin{aligned}
\int_{-\infty}^{\infty} s(t) s^{\prime \prime}(t) d t & =\frac{1}{2 \pi} \int_{-\infty}^{\infty} S(\Omega)\left(-\Omega^{2}\right) S^{*}(\Omega) d \Omega \\
& =-\frac{1}{2 \pi} \int_{-\infty}^{\infty} \Omega^{2}|S(\Omega)|^{2} d \Omega \\
& =-\int_{-\infty}^{\infty} s^{\prime}(t) s^{\prime}(t) d t<0
\end{aligned}
$$

where $S(\Omega)$ denotes the Fourier transform of $s(t)$; Nyquist sampling is assumed.

Assuming that $x_{i}(t)$ in (9) is sampled at the Nyquist rate, orthogonality applies also to the sampled counterparts $\mathbf{s}, \mathbf{s}^{\prime}$, and $\mathbf{s}^{\prime \prime}$, and $E_{s s^{\prime \prime}}=\mathbf{s}^{T} \mathbf{s}^{\prime \prime}=-E_{s^{\prime}}$.

Using these observations, we can see that the first eigenvector of the correlation matrix in (10) should be a linear combination between $\mathbf{s}$ and $\mathbf{s}^{\prime \prime}$ of the form $\left(\mathbf{s}+\alpha \mathbf{s}^{\prime \prime}\right)$, where $\alpha$ is a scale factor to be determined. When multiplying $\mathbf{s}$ and $\mathbf{s}^{\prime \prime}$ with the term 
being a combination of $\mathbf{s}$ and $\mathbf{s}^{\prime \prime}$ in $\mathbf{R}_{x}$, we obtain

$$
\left(\mathbf{s s}^{T}+\frac{\sigma_{\theta}^{2}}{2}\left(\mathbf{s s}^{\prime \prime T}+\mathbf{s}^{\prime \prime} \mathbf{s}^{T}\right)\right) \mathbf{s}=\underbrace{\left(E_{s}-\frac{\sigma_{\theta}^{2}}{2} E_{s^{\prime}}\right)}_{C_{s s}} \mathbf{s}+\underbrace{\left(\frac{\sigma_{\theta}^{2}}{2} E_{s}\right)}_{C_{s s^{\prime \prime}}} \mathbf{s}^{\prime \prime}
$$

and

$$
\left(\mathbf{s s}^{T}+\frac{\sigma_{\theta}^{2}}{2}\left(\mathbf{s s}^{\prime \prime T}+\mathbf{s}^{\prime \prime} \mathbf{s}^{T}\right)\right) \mathbf{s}^{\prime \prime}=\underbrace{\left(-E_{s^{\prime}}+\frac{\sigma_{\theta}^{2}}{2} E_{s^{\prime \prime}}\right)}_{C_{s^{\prime \prime} s}} \mathbf{s}+\underbrace{\left(-\frac{\sigma_{\theta}^{2}}{2} E_{s^{\prime}}\right)}_{C_{s^{\prime \prime}} s^{\prime \prime}} \mathbf{s}^{\prime \prime} .
$$

Thus, the eigenvalue equation for $\lambda_{1}$, for convenience expressed as

$$
\lambda_{1}=\lambda_{1, s}+\sigma_{v}^{2},
$$

is given by $\mathbf{R}_{x}\left(\mathbf{s}+\alpha \mathbf{s}^{\prime \prime}\right)=\left(\lambda_{1, s}+\sigma_{v}^{2}\right)\left(\mathbf{s}+\alpha \mathbf{s}^{\prime \prime}\right)$, yielding

$$
C_{s s} \mathbf{s}+C_{s s^{\prime \prime}} \mathbf{s}^{\prime \prime}+\alpha\left(C_{s^{\prime \prime} s} \mathbf{s}+C_{s^{\prime \prime} s^{\prime \prime}} \mathbf{s}^{\prime \prime}\right)=\lambda_{1, s}\left(\mathbf{s}+\alpha \mathbf{s}^{\prime \prime}\right) .
$$

To estimate the eigenvalue, the following equation system should be solved:

$$
\begin{gathered}
C_{s s}+\alpha C_{s^{\prime \prime} s}=\lambda_{1, s}, \\
C_{s s^{\prime \prime}}+\alpha C_{s^{\prime \prime} s^{\prime \prime}}=\alpha \lambda_{1, s} .
\end{gathered}
$$

Solving for $\lambda_{1, s}$ the following quadratic equation results:

$$
\lambda_{1, s}^{2}-\lambda_{1, s}\left(C_{s^{\prime \prime} s^{\prime \prime}}+C_{s s}\right)+\left(C_{s s} C_{s^{\prime \prime} s^{\prime \prime}}-C_{s s^{\prime \prime}} C_{s^{\prime \prime} s}\right)=0,
$$

whose solutions are given by

$$
\begin{aligned}
\lambda_{1, s} & =\frac{C_{s^{\prime \prime} s^{\prime \prime}}+C_{s s} \pm \sqrt{\left(C_{s^{\prime \prime} s^{\prime \prime}}+C_{s s}\right)^{2}-4\left(C_{s s} C_{s^{\prime \prime} s^{\prime \prime}}-C_{s s^{\prime \prime}} C_{s^{\prime \prime} s}\right)}}{2} \\
& =\frac{C_{s^{\prime \prime} s^{\prime \prime}}+C_{s s} \pm \sqrt{\left(C_{s^{\prime \prime} s^{\prime \prime}}-C_{s s}\right)^{2}+4 C_{s s^{\prime \prime}} C_{s^{\prime \prime} s}}}{2} .
\end{aligned}
$$

Substituting the $C$ coefficients defined in (37) and (38), we obtain

$$
\lambda_{1, s}=\frac{E_{s}-\sigma_{\theta}^{2} E_{s^{\prime}} \pm \sqrt{E_{s}^{2}+4\left(-E_{s^{\prime}}+\frac{\sigma_{\theta}^{2}}{2} E_{s^{\prime \prime}}\right) \frac{\sigma_{\theta}^{2}}{2} E_{s}}}{2} .
$$


Approximating the square root, realizing that higher-order terms are always smaller than the lower-order terms for small $\sigma_{\theta}^{2}$, and retaining the positive sign of the square root solution, we obtain

$$
\lambda_{1, s} \approx E_{s}-\frac{\sigma_{\theta}^{2}}{2} E_{s^{\prime}}+\left(-E_{s^{\prime}}+\frac{\sigma_{\theta}^{2}}{2} E_{s^{\prime \prime}}\right) \frac{\sigma_{\theta}^{2}}{2} .
$$
smaller eigenvalue. Neglecting the fourth-order term, we obtain

$$
\lambda_{1, s} \approx E_{s}-\sigma_{\theta}^{2} E_{s^{\prime}}
$$

which, when substituted in (39), becomes the desired eigenvalue in (11), i.e.,

$$
\lambda_{1} \approx E_{s}-\sigma_{\theta}^{2} E_{s^{\prime}}+\sigma_{v}^{2} .
$$

The $\alpha$ factor in the linear combination between $\mathbf{s}$ and $\mathbf{s}^{\prime \prime}$, using the above approximations, results in $\alpha=\sigma_{\theta}^{2} / 2$. Hence, the first eigenvector $\psi_{1}$ is proportional to ${ }_{525}\left(\mathbf{s}+\left(\sigma_{\theta}^{2} / 2\right) \mathbf{s}^{\prime \prime}\right)$ as expressed in (12).

Repeating the same derivation for $\hat{\mathbf{R}}_{x}^{\bullet}$ in (19), $\boldsymbol{\psi}_{1}^{\bullet}$ should be proportional to the form $\left(\mathbf{1}+\alpha^{\bullet} \boldsymbol{\theta}^{2}\right)$, and the equations corresponding to (37) and (38) become:

$$
\frac{1}{N}\left(E_{s} \mathbf{1} \mathbf{1}^{T}-\frac{E_{s^{\prime}}}{2}\left(\mathbf{1} \boldsymbol{\theta}^{2^{T}}+\boldsymbol{\theta}^{2} \mathbf{1}^{T}\right)\right) \mathbf{1}=\underbrace{\frac{M}{N}\left(E_{s}-\frac{\sigma_{\theta}^{2}}{2} E_{s^{\prime}}\right)}_{C_{s s}} \mathbf{1}+\underbrace{\left(-\frac{M}{2 N} E_{s^{\prime}}\right)}_{C_{s s^{\prime \prime}}} \boldsymbol{\theta}^{2}
$$

and

$\frac{1}{N}\left(E_{s} \mathbf{1 1}^{T}-\frac{E_{s^{\prime}}}{2}\left(\mathbf{1}^{2^{T}}+\boldsymbol{\theta}^{2} \mathbf{1}^{T}\right)\right) \boldsymbol{\theta}^{2}=\underbrace{\frac{M}{N}\left(\sigma_{\theta}^{2} E_{s}-\frac{3 \sigma_{\theta}^{4}}{2} E_{s^{\prime}}\right)}_{C_{s^{\prime \prime}}} \mathbf{1}+\underbrace{\left(-\frac{M \sigma_{\theta}^{2}}{2 N} E_{s^{\prime}}\right)}_{C_{s^{\prime \prime} s^{\prime \prime}}} \boldsymbol{\theta}^{2}$,

yielding

$$
\lambda_{1}^{\bullet} \approx \frac{E_{s} M}{N}-\frac{\sigma_{\theta}^{2} E_{s^{\prime}} M}{N}+\sigma_{v}^{2}
$$

which is the desired eigenvalue in (20). Deriving $\alpha^{\bullet}$ using the above approximations, we obtain that $\alpha^{\bullet}=-E_{s^{\prime}} /\left(2 E_{s}\right)$, leading to the eigenvectors in (21). 


\section{Appendix B}

This appendix derives the expression for $\hat{\boldsymbol{\theta}}_{\mathrm{ML}}$. First, we observe that for the model in (1) the probability density function (PDF) of $x_{i}(n)$, given a sample $n$, a deterministic

Acknowledgment The authors want to acknowledge Javier Preciado for an early implementation of the one-step estimate normalization factor and Juan Pablo Martínez for valuable comments on the manuscript. This work was supported by projects DPI201675458-R, DPI2015-68820-R funded by MINECO and FEDER; by Gobierno de Aragón 
and European Social Fund (EU) through Biomedical Signal Interpretation and Com-

putational Simulation (BSICoS) Group (T96); by CERCA Programme / Generalitat de Catalunya and by CIBER in Bioengineering, Biomaterials \& Nanomedicne (CIBERBBN) through Instituto de Salud Carlos III and FEDER (Spain). The computation was performed by the ICTS NANBIOSIS, specifically by the High Performance Computing Unit of the CIBER-BBN at the University of Zaragoza.

\section{Reference}

[1] S. M. Kay, Fundamentals of Statistical Signal Processing. Estimation Theory, Prentice-Hall, New Jersey, 1993.

[2] L. Sörnmo, P. Laguna, Bioelectrical Signal Processing in Cardiac and Neurological Applications, Elsevier (Academic Press), Amsterdam, 2005.

[3] K. J. Coakley, P. Hale, Alignment of noisy signals, IEEE Trans. Instrum. Measure. 50 (2001) 141-149, doi:10.1109/19.903892.

[4] S. Gibson, J. W. Judy, D. Markovic, Spike sorting: The first step in decoding the brain, IEEE Signal Process. Mag. 29 (2012) 124-143, doi:10.1109/MSP.2011.941880.

[5] W. Muhammad, O. Meste, H. Rix, Comparison of single and multiple time delay estimators: Application to muscle fiber conduction velocity estimation, Signal Process. 82 (2002) 925-940, doi:10.1016/S0165-1684(02)00202-5.

[6] A. Cabasson, O. Meste, G. Blain, S. Bermon, Quantifying the PR interval pattern during dynamic exercise and recovery, IEEE Trans. Biomed. Eng. 56 (2009) 2675-2683, doi:10.1109/TBME.2009.2028694.

[7] A. Cabasson, O. Meste, J. M. Vesin, Estimation and modeling of QT-interval adaptation to heart rate changes, IEEE Trans. Biomed. Eng. 59 (2012) 956-565, doi:10.1109/TBME.2011.2181507. 
[8] R. Jané, H. Rix, P. Caminal, P. Laguna, Alignment methods for averaging of high resolution cardiac signals: A comparative study of performance, IEEE Trans. Biomed. Eng. 38 (1991) 571-579, doi:10.1109/10.81582.

[9] W. Truccolo, K. H. Knuth, A. Shah, S. L. Bressler, C. E. Schroeder, M. Ding, Estimation of single-trial multicomponent ERPs: differentially variable component analysis (dVCA), Biol. Cybern. 89 (2003) 426-438, doi:10.1007/s00422003-0433-7.

[10] A. Zviagintsev, Y. Perelman, R. Ginosar, Algorithms and architectures for low power spike detection and alignment, J. Neural Eng. 3 (2006) 35-42, doi:10.1088/1741-2560/3/1/004.

[11] K. C. McGill, L. J. Dorfman, High-resolution alignment of sampled waveforms, IEEE Trans. Biomed. Eng. 31 (1984) 462-468, doi:10.1109/TBME.1984.325413.

[12] D. T. Pham, J. Möcks, W. Köhler, T. Gasser, Variable latencies of noisy signals: Estimation and testing in brain potential data, Biometrika 74 (1987) 525-533, doi:10.1093/biomet/74.3.525.

[13] P. Jáskowski, R. Verleger, Amplitudes and latencies of single-trial ERP's estimated by a maximum-likelihood method, IEEE Trans. Biomed. Eng. 46 (1999) 987-993, doi:10.1109/10.775409.

[14] D. Farina, W. Muhammad, E. Fortunato, O. Meste, R. Merletti, H. Rix, Estimation of single motor unit conduction velocity from surface electromyogram signals detected with linear electrode arrays, Med. Biol. Eng. \& Comput 39 (2001) 225-236, doi:10.1007/BF02344807.

[15] E. Laciar, R. Jané, D. H. Brooks, Improved alignment method for noisy highresolution ECG and Holter records using multiscale cross-correlation, IEEE Trans. Biomed. Eng. 50 (2003) 344-353, doi:10.1109/TBME.2003.808821.

[16] C. D. Woody, Characterization of an adaptive filter for the analysis of variable latency neuroelectric signals, Med. Biol. Eng. 5 (1967) 539-553. 
[17] G. H. Steeger, O. Hermann, M. Spreng, Some improvements in the measurement of variable latency acoustically evoked potentials in human EEG, IEEE Trans. Biomed. Eng. 30 (1983) 295-303, doi:10.1109/TBME.1983.325119.

[18] D. H. Lange, H. Pratt, G. F. Inbar, Modeling and estimation of single evoked brain potential components, IEEE Trans. Biomed. Eng. 44 (1997) 791-799, doi:10.1109/10.623048.

[19] L. Xu, P. Stoica, J. Li, S. L. Bressler, X. Shao, M. Ding, ASEO: A method for the simultaneous estimation of single-trial event-related potentials and ongoing brain activities, IEEE Trans. Biomed. Eng. 56 (2009) 111-121, doi:10.1109/TBME.2008.2008166.

[20] L. Gupta, D. L. Molfese, R. Tammana, P. G. Simos, Nonlinear alignment and averaging for estimating the evoked potential, IEEE Trans. Biomed. Eng. 43 (1996) 348-356, doi:10.1109/10.486255.

[21] S. Casarotto, A. M. Bianchi, S. Cerutti, G. A. Chiarenza, Dynamic time warping in the analysis of event-related potentials, IEEE Eng. Med. Biol. Mag. 24 (2005) 68-77, doi:10.1109/MEMB.2005.1384103.

[22] A. Cabasson, O. Meste, Time delay estimation: A new insight into the Woody's method, IEEE Signal Process. Letters 15 (2008) 573-576, doi:10.1109/LSP.2008.2001558.

[23] K. Kim, S. H. Lim, J. Lee, W. S. Kang, C. Moon, J. W. Choi, Joint Maximum Likelihood Time Delay Estimation of Unknown Event-Related Potential Signals for EEG Sensor Signal Quality Enhancement, Sensors 16, 891(2016) 1-17, doi:10.3390/s16060891

[24] A. Garde, L. Sörnmo, P. Laguna, R. Jané, S. Benito, A. Bayes-Genis, B. Giraldo, Assessment of Respiratory Flow Cycle Morphology in Patients with Chronic Heart Failure, Med. Biol. Eng. \& Comput 55 (2017) 245-255, doi:10.1007/s11517-016-1498-5. 
[25] F. Castells, P. Laguna, L. Sörnmo, A. Bollmann, J. Millet Roig, Principal component analysis in ECG signal processing, J. Adv. Signal Process. (www.hindawi.com/journals/asp) 2007 (ID 74580), doi:10.1155/2007/74580.

[26] P. Laguna, L. Sörnmo, Sampling rate and the estimation of ensemble variability for repetitive signals, Med. Biol. Eng. \& Comput. 38 (2000) 540-546, doi:10.1007/BF02345750. doi:10.1007/BF02345750.

[27] F. Van den Bergh, A. P. Engelbrecht, A cooperative approach to particle swarm optimization, IEEE Trans. Evol. Comput. 8 (2004) 225-239, doi:10.1109/TEVC.2004.826069.

[28] B. Niu, Y. Zhu, X. He, H. Wu, MCPSO: A multi-swarm cooperative particle swarm optimizer, Appl. Math. Comput. 2 (2007) 1050-1062, doi:10.1016/j.amc.2006.07.026.

[29] A. Garde, L. Sörnmo, R. Jané, B. F. Giraldo, Correntropy-based spectral characterization of respiratory patterns in patients with chronic heart failure, IEEE Trans. Biomed. Eng. 57 (2010) 1964-1972, doi: 10.1109/TBME.2010.2044176.

[30] S. Abboud, R. J. Cohen, A. Selwyn, P. Ganz, D. Sadeh, P. L. Friedman, Detection of transient myocardial ischemia by computer analysis of standard and signal-averaged high-frequency electrocardiograms in patients undergoing percutaneous transluminal coronary angioplasty, Circulation 76 (1987) 585-596, doi:10.1161/01.CIR.76.3.585.

[31] J. Pettersson, G. Wagner, L. Sörnmo, E. Trägårdh-Johansson, H. Öhlin, O. Pahlm, High-frequency ECG as a supplement to standard 12-lead ischemia monitoring during reperfusion therapy of acute inferior myocardial infarction, J. Electrocardiol. 44 (2011) 11-17, doi:10.1016/j.jelectrocard.2010.04.006.

[32] F. Marini, B. Walczak, Particle swarm optimization (PSO). A tutorial, Chemometr. Intell. Lab. Syst. 149 (2015) 153-165, doi:10.1016/j.chemolab.2015.08.020. 


\section{Biography}

Pablo Laguna is Professor of Signal Processing and Communications and researcher at the Aragón Institute for Engineering Research (I3A), both at University of Zaragoza, Spain. He is also member of the Spanish Research Center CIBER-BBN. $\mathrm{He}$ is President of Computing in Cardiology, and Associate Editor of Digital Signal Processing and Medical and Biological Engineering \& Computing. He is also responsible of the Ph.D. program in BME at University of Zaragoza.

Ainara Garde is Assistant Professor at Department of Biomedical Signals and Systems (BSS), University of Twente, Enschede, The Netherlands. She was a postdoctoral fellow at the University of British Columbia (BC) and BC Childrens Hospital, Canada. She received the Ph.D. degree in biomedical engineering from the Technical University of Catalonia, Spain, in 2010.

Beatriz F. Giraldo is Associate Professor of Automatic Control and Statistical Analysis of Biomedical Signal Processing at Technical University of Catalonia (UPC), and is serving as Academic Secretary of the Barcelona East School of Engineering. She is Senior Researcher at Biomedical Signal Processing and Interpretation (BIOSPIN), IBEC and CIBER-BBN, Spain.

Olivier Meste is Professor at the Computer Science, Signals and Systems Laboratory of Sophia Antipolis (I3S), University of Nice, France. He is Head of Department of Electrical Engineering and Industrial Data Processing at Institut Universitaire de Technologie (IUT) and biomedical signal processing research at the I3S laboratory. He was member of the Bio Imaging and Signal Processing technical committee of the IEEE Signal Processing Society (2006-2012).

Raimon Jané is Director of Research in the Department of Automatic Control (ESAII), UPC, and the Scientific Group Leader of the Biomedical Signal Processing and Interpretation Group, Institute for Bioengineering of Catalonia (IBEC), Barcelona. $\mathrm{He}$ is the Principal Investigator of the Biomedical Signals and Systems (SISBIO) Group of the Biomedical Research Networking Center in Bioengineering, Biomaterials and Nanomedicine (CIBER-BBN), Spain. He is President of the Spanish Society of BME and coordinator of Ph.D. program in BME at UPC University. 
Leif Sörnmo is Professor of Biomedical Signal Processing and Head of the Biomedical Signal Processing Group, Department of Biomedical Engineering, Lund University. He serves on the board of Computing in Cardiology. He is Associate Editor of IEEE Transactions of Biomedical Engineering and Medical and Biological Engineering \& Computing. Dr. Sörnmo is a Fellow of the IEEE, International Academy of Medical and Biological Engineering, and European Alliance for Medical and Biological Engineering. 